\title{
Antimicrobial Effects of Novel Triple Antibiotic Paste-Mimic Scaffolds on Actinomyces naeslundii Biofilm
}

\author{
Maria T.P. Albuquerque, DDS, MSD, ${ }^{* \dagger}$ Stuart J. Ryan, BS, ${ }^{\star}$ Eliseu A. Münchow, DDS, MSD, ${ }^{*}$ \\ Maria M. Kamocka, PhD, ${ }^{\downarrow}$ Richard L. Gregory, PhD, Marcia C. Valera, DDS, MSD, ${ }^{\dagger}$ \\ and Marco C. Bottino, DDS, MSc, PhD*
}

\section{Ahstract}

Introduction: Actinomyces naes/undii has been recovered from traumatized permanent teeth diagnosed with necrotic pulps. In this work, a triple antibiotic paste (TAP)-mimic scaffold is proposed as a drug-delivery strategy to eliminate $A$. naes/undii dentin biofilm. Methods: Metronidazole, ciprofloxacin, and minocycline were added to a polydioxanone (PDS) polymer solution and spun into fibrous scaffolds. Fiber morphology, mechanical properties, and drug release were investigated by using scanning electron microscopy, microtensile testing, and high-performance liquid chromatography, respectively. Human dentin specimens (4 $\times 4 \times 1 \mathrm{~mm}^{3}, n=4 /$ group) were inoculated with A. naes/undii (ATCC 43146) for 7 days for biofilm formation. The infected dentin specimens were exposed to TAP-mimic scaffolds, TAP solution (positive control), and pure PDS (drug-free scaffold). Dentin infected (7-day biofilm) specimens were used for comparison (negative control). Confocal laser scanning microscopy was done to determine bacterial viability. Results: Scaffolds displayed a submicron mean fiber diameter (PDS = $689 \pm 312 \mathrm{~nm}$ and TAP-mimic $=718 \pm 125 \mathrm{~nm})$. Overall, TAP-mimic scaffolds showed significantly $(P \leq .040)$ lower mechanical properties than PDS. Within the first 24 hours, a burst release for all drugs was seen. A sustained maintenance of metronidazole and ciprofloxacin was observed over 4 weeks, but not for minocycline. Confocal laser scanning microscopy demonstrated complete elimination of all viable bacteria exposed to the TAP solution. Meanwhile, TAP-mimic scaffolds led to a significant $(P<.05)$ reduction in the percentage of viable bacteria compared with the negative control and PDS. Conclusions: Our findings suggest that TAPmimic scaffolds hold significant potential in the eradication/elimination of bacterial biofilm, a critical step in regenerative endodontics. (J Endod 2015;41:13371343)

\section{Key Words}

Antibiotic, bacteria, disinfection, electrospinning, nanofibers, pulp, regeneration, root canal, scaffold, stem cells

$R$ egeneration of the pulp-dentin complex is highly dependent on the effective elimiInation of bacterial overgrowth within the root canal system $(1,2)$. Several microbial species have been identified inside root canals, including but not limited to Actinomyces naeslundii. A gram-positive filamentous, rod-shaped facultative anaerobe commonly found in the gastrointestinal tract (3), A. naeslundii has been increasingly correlated to the ability of biofilm formation and to failed endodontic therapy (4-6). Recently, A. naeslundii was detected in initial samples recovered from 10 of 15 traumatized permanent immature teeth diagnosed with necrotic pulps (7), suggesting the species may be relevant to regenerative endodontics.

The traditionally advocated substances used in endodontics to combat root canal infection, including sodium hypochlorite and calcium hydroxide, have shown ineffectiveness, mainly in cases of biofilm-related persistent infections $(8,9)$. In recent years, antibiotic mixtures such as triple antibiotic paste (TAP), a mixture of metronidazole (MET), ciprofloxacin (CIP), and minocycline (MINO) (10, 11), has gained increased evidence because of its clinically proven disinfection role within the revascularization strategy $(12,13)$. Regardless of the promising clinical evidence of pulp-like tissue regeneration, TAP uses a considerably high concentration $(1 \mathrm{~g} / \mathrm{mL})$ of these antibiotics, which has demonstrated harmful effects on cell viability/proliferation $(14,15)$.

In light of this, the use of antibiotic-containing scaffolds as drug-delivery systems has demonstrated significant clinical potential $(2,16)$ when compared with TAP from a cytocompatibility standpoint. To our knowledge, no attempt has been made to develop and establish an $A$. naeslundii biofilm on dentin and understand its susceptibility to antimicrobial substances used in regenerative endodontics. We report for the first time the antimicrobial effects of a novel TAP-mimic scaffold on $A$. naeslundii-infected human dentin biofilm by using confocal laser scanning microscopy (CLSM).

\section{Materials and Method \\ Synthesis of TAP-mimic Electrospun Polymer Scaffolds}

Polydioxanone (PDS II; Ethicon, Somerville, NJ) filaments were subjected to an undying process to remove the violet color. In brief, PDS filaments were immersed in dichloromethane (Sigma-Aldrich, St Louis, M0) for 2 days (16-19). A 10 wt\% PDS solution was prepared in 1,1,1,3,3,3-hexafluoro-2-propanol (Sigma-Aldrich).

From the *Division of Dental Biomaterials, Department of Restorative Dentistry, ${ }^{\S}$ Division of Nephrology, and "Department of Oral Biology, Indiana University School of Dentistry, Indianapolis, Indiana; ${ }^{\dagger}$ Graduate Program in Restorative Dentistry (Endodontics), Universidade Estadual Paulista, São José dos Campos Dental School, São José dos Campos, São Paulo, Brazil; and ${ }^{\ddagger}$ Department of Operative Dentistry, Federal University of Pelotas (UFPEL), School of Dentistry, Pelotas, Rio Grande do Sul, Brazil.

Address requests for reprints to Dr Marco Cicero Bottino, Division of Dental Biomaterials, Department of Restorative Dentistry, Indiana University School of Dentistry, 1121 West Michigan Street (DS112A), Indianapolis, IN 46202. E-mail address: mbottino@iu.edu

0099-2399/\$ - see front matter

Copyright (C) 2015 American Association of Endodontists.

http://dx.doi.org/10.1016/j.joen.2015.03.005 
MET, CIP, and MINO (Sigma-Aldrich) were added to the polymer solution at $25 \mathrm{wt} \%$ concentration (relative to the total PDS [600 mg] weight, ie, $150 \mathrm{mg}$ of each drug) and mixed together under stirring (16-19). Pure PDS (control) and the TAP-mimic polymer solutions were spun into scaffolds (ie, flow rate $2 \mathrm{~mL} / \mathrm{h}, 18-\mathrm{cm}$ distance, and electrical voltage between 15 and $19 \mathrm{kV}$ ). The polymer solutions were individually placed into plastic syringes (Becton, Dickinson and Company, Franklin Lakes, NJ) fitted with a metallic 27-gauge blunt needle, and the fibers were collected on an aluminum foil covered rotating mandrel at room temperature (RT) (16-19). To ensure complete elimination of any residual solvent, the scaffolds were dried for 48 hours under vacuum at $\mathrm{RT}$ and then stored at $4^{\circ} \mathrm{C}(16-19)$.

\section{Fiher Morphology, Mechanical Characterization, and Drug Release}

Fiber morphology of the scaffolds was investigated under a scanning electron microscope (FE-SEM, Model JSM-6701F; JEOL, Tokyo, Japan). The fiber diameter was measured (Image-J; National Institutes of Health, Bethesda, MD). The scaffolds' mechanical properties were determined by tensile testing (expert 5601; ADMET, Norwood, MA) of specimens $\left(15 \times 3 \mathrm{~mm}^{2}, n=10 /\right.$ group/condition $)$ under dry and wet conditions (24-hour incubation in phosphate-buffered saline [PBS]) $(16,17)$.

Evaluation of the drug release from TAP-mimic scaffolds $(15 \times 15$ $\left.\mathrm{mm}^{2}, n=4\right)$ was done via high-performance liquid chromatography (HPLC). The scaffolds were weighed and then immersed in PBS (10 $\mathrm{mL}$ ) for 28 days. Aliquots $(1 \mathrm{~mL})$ were removed from each solution at selected time points $(1,3,7,14$, and 28 days) and replaced with an equal amount of fresh PBS at each collection $(16,18)$. Aliquots $(10 \mu \mathrm{L})$ were analyzed by using HPLC-UV equipment (Agilent 1100 System, Palo Alto, CA; Zorbax SB-phenyl chromatography column), which consisted of a binary mobile phase of solvent systems A $(0.1 \%$ formic acid in $\left.\mathrm{ddH}_{2} \mathrm{O}, \mathrm{v} / \mathrm{v}\right)$ and $\mathrm{B}(0.1 \%$ formic acid in acetonitrile, $\mathrm{v} / \mathrm{v})$ in a gradient elution. Test parameters were described in detail elsewhere $(16,18)$. The percentage of the released drugs was then calculated on the basis of the initial weight of the scaffolds $(16,18)$.

\section{Antimicrobial Evaluation of A. naes/undii Biofilm-infected Dentin Specimens}

Caries-free human mandibular canines were collected under an approved (protocol \#1407656657) local Institutional Review Board protocol (Indiana University). Teeth were washed, and the soft tissue remains were removed before storage in thymol $0.1 \%$. After crown sectioning by using a diamond disk, the teeth were sectioned along the buccolingual plane, obtaining 2 halves for dentin specimen $\left(4 \times 4 \times 1 \mathrm{~mm}^{3}\right)$ preparation. The cementum was removed, and the specimens were wet-finished with $\mathrm{SiC}$ papers (600-1200 grit). All the specimens were immersed in 2.5\% NaOCl and 17\% EDTA (Inter-Med, Inc, Racine, WI) for 3 minutes each in an ultrasonic bath (L\&R 2014 Ultrasonic Cleaning System; L\&R Manufacturing Company, Keamy, NJ) to remove the smear layer (19). Last, the specimens were rinsed in saline solution for 10 minutes, sterilized in an autoclave $\left(121^{\circ} \mathrm{C}\right.$ for 20 minutes), and then randomly placed with the dentin side positioned upward in 24-well plates (Costar; Corning Life Sciences, Tewksbury, MA) before A. naeslundii (ATCC 43146) inoculation. In brief, $200 \mu \mathrm{L}$ of 16- to 18-hour culture of the bacterial suspension (ca. $10^{6}$ bacteria) was pipetted into each well containing $1.8 \mathrm{~mL}$ brain-heart infusion medium (Difco Laboratories Inc, Detroit, MI). The plates were kept $\left(37^{\circ} \mathrm{C}\right)$ aerobically in an incubator for 7 days to allow biofilm formation. The broth was changed every other day. All the specimens were rinsed for 1 minute $(2 \times)$ with PBS (Sigma-Aldrich) to remove nonadherent bacterial cells before treatment. Infected dentin specimens ( $n=6$ /group) were randomly allocated to 3 experimental groups: pure PDS scaffolds (drug-free), TAP-mimic scaffolds $(13.15 \pm 0.3 \mathrm{mg}$, ca. $3.3 \mathrm{mg}$ of each antibiotic), TAP solution (50 $\mathrm{mg} / \mathrm{mL}$ of each of the drugs), and the negative control group (7-day biofilm untreated). The scaffolds $\left(15 \times 15 \mathrm{~mm}^{2}\right)$ were sterilized by ultraviolet irradiation (16-19) adapted to plastic inserts (CellCrown; Scaffdex Ltd, Tampere, Finland) and then placed into the wells containing the infected dentin specimens and $1 \mathrm{~mL}$ PBS (19). The plates were incubated under aerobic conditions at $37^{\circ} \mathrm{C}$ for 3 days, and the specimens were prepared for CLSM. Finally, the dentin specimens were rinsed in PBS $(2 \times)$ to remove unbound bacteria and medium. Two specimens per group were prepared for scanning electron microscopy (SEM) imaging. The remaining specimens in each group were stained with the fluorescent LIVE/DEAD BacLight Bacterial Viability Kit L-7012 (Molecular Probes, Eugene, OR) containing SYTO 9 and propidium iodide (PI). The dyes were mixed in a 1:1 solution and applied to the specimens for 30 minutes at RT before CLSM analysis. Live bacteria presenting intact cell membranes were dyed green (SYTO 9), whereas dead bacteria with damaged membranes were stained red (PI) (20-23). A total of 16 dentin specimens $(n=4$ /group) and 5 randomly selected microscopic fields were scanned, starting from the edges of the specimen to obtain 20 measurements per group. Images were acquired with a confocal/2-photon Leica TCS SP8 system (Leica Microsystems Inc, Buffalo Grove, IL) by using Leica HC PL APO $40 \times / 1.3$ oil immersion objective. Series of sections through the depth of tissue (Z-stacks) were collected by using optimal step size settings $(0.35 \mu \mathrm{m})$; images were composed of $512 \times 512$ pixels $\left(221 \times 221 \mu \mathrm{m}^{2}\right)$. Data quantification and 3-dimensional volume reconstruction were performed by using dedicated software (Imaris 7.7; Bitplane USA, South Windsor, CT) (23, 24). Volume images were processed to extract a statistical parameter of live and dead bacteria volume. Data were presented as a ratio of LIVE/DEAD bacteria.

\section{Statistical Analysis}

The mechanical data were analyzed (SigmaPlot version 12; Systat Software Inc, San Jose, CA) by using two-way analysis of variance and Tukey test for multiple comparison $(\alpha=0.05)$. For CLSM analysis, groups were compared for differences in the percentage of dead bacterial cells by using the mixed-model analysis of variance, with a fixed effect for group and a random effect for sample, to account for measurements at multiple areas on each specimen. A variance stabilizing transformation $\left(\sin ^{-1}[(\right.$ percent dead $) 1 / 2)$ was used to satisfy the assumptions.

\section{Results Fiher Morphology, Mechanical Gharacterization, and Drug Release}

A submicron mean fiber diameter was seen for both pure PDS $(689 \pm 312 \mathrm{~nm})$ and TAP-mimic $(718 \pm 125 \mathrm{~nm})$ scaffolds (Fig. $1 A$ and $B$ ). Whereas PDS scaffolds exhibited a wider fiber diameter distribution (Fig. 1C), the TAP-mimic scaffold presented a gaussian distribution (Fig. 1D), with most of the fibers ranging from 600 to $800 \mathrm{~nm}$. Mechanical performances of the bioactive scaffolds are displayed in Figure $1 E-G$. Data analysis revealed that the scaffold $(P=.020)$ and storage condition factors $(P \leq .001)$ were both significant, although their interaction was not $(P=.541)$. Figure 2 reveals a burst release of the drugs within the first 24 hours, which was sustained for MET and CIP but not for MINO, which displayed a progressive concentration reduction within 7 days of incubation. 

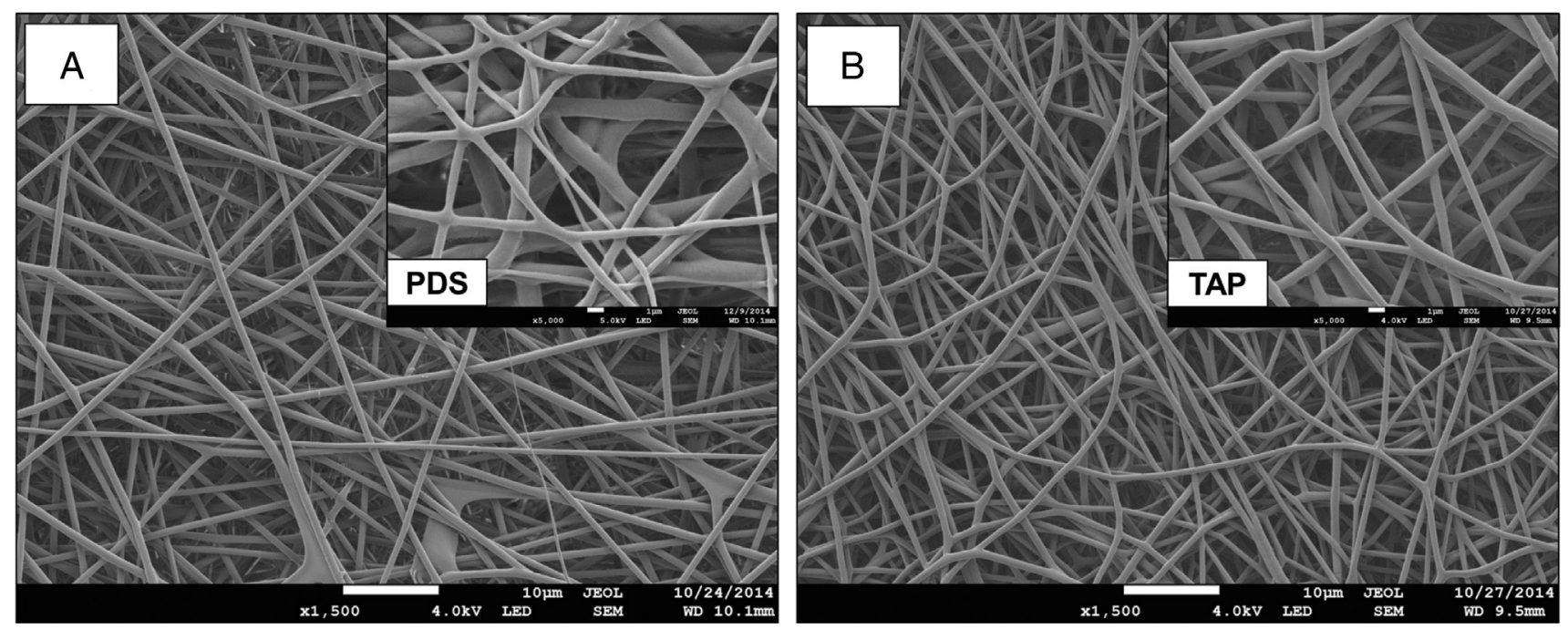

C

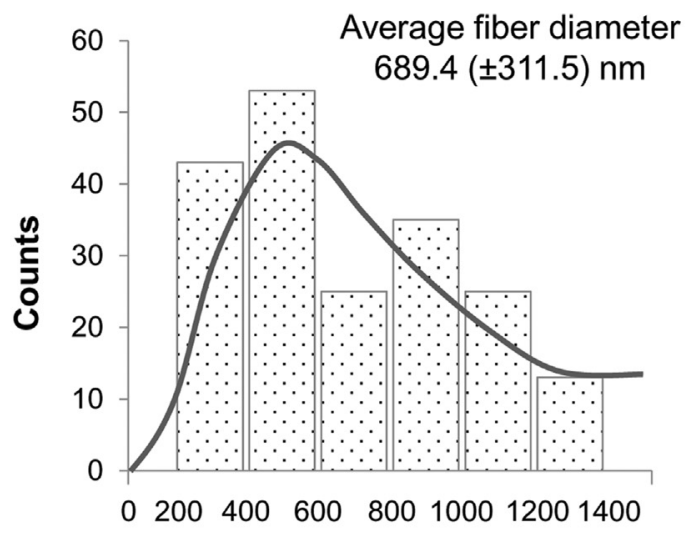

Fiber diameter $(\mathbf{n m})$
$\mathrm{D}$

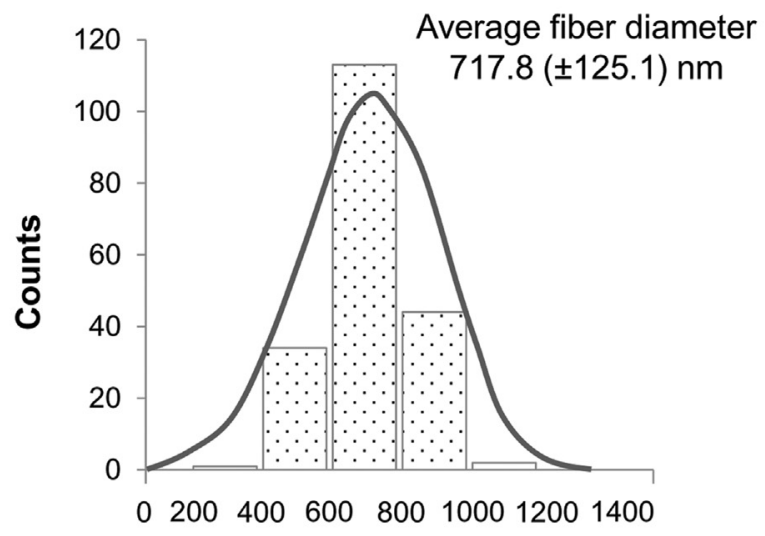

Fiber diameter (nm)
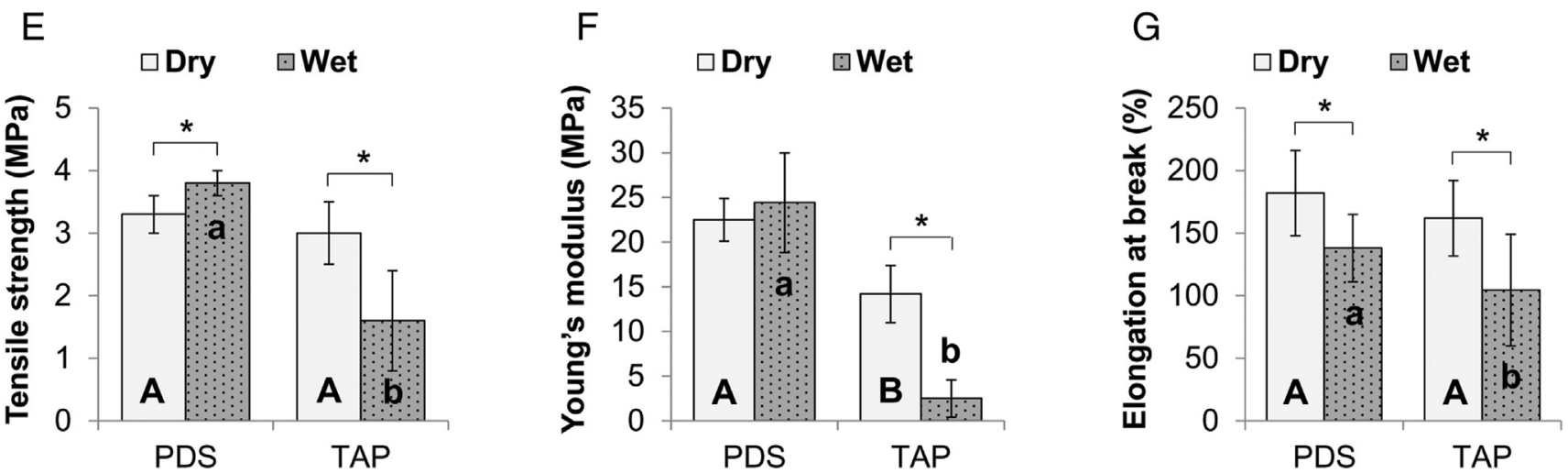

Figure 1. SEM micrographs (original magnification, $\times 1500$ and $\times 5000)$ of pure PDS $(A)$ and TAP-mimic $(B)$ scaffolds obtained via electrospinning (processing parameters used: needle-collector distance of $18 \mathrm{~cm}$, flow rate of $2 \mathrm{~mL} \cdot \mathrm{h}^{-1}$, and voltage of $\left.15-19 \mathrm{kV}\right)$. Both scaffolds presented a porous structure and smooth fibers with similar average fiber diameter, although the former $(C)$ showed a wider range of diameter distribution (heterogeneous) compared with the latter $(D)$, which displayed a gaussian distribution (homogeneous). Regarding the mechanical analysis (ie, tensile strength $[E]$, Young's modulus $[F]$, and elongation at break $[G]$ ), statistically significant differences between drug-free scaffolds (pure PDS) and TAP-mimic scaffolds were seen (represented by different uppercase [dry condition] and lowercase [wet condition] letters $[P<.05]$ ). In detail, under dry conditions, the TAP-mimic scaffold demonstrated similar tensile strength $(P=.133)$ and elongation at break $(P=.200)$ when compared with pure PDS, although presenting with a lower Young's modulus $(P<.001)$. After hydration, lower mechanical properties $(P \leq .040)$ were obtained for the TAP-mimic scaffold when compared with the control. Asterisk $\left(^{*}\right)$ above bar columns indicates statistically significant difference between dry and wet storage conditions $(P<.05)$. 


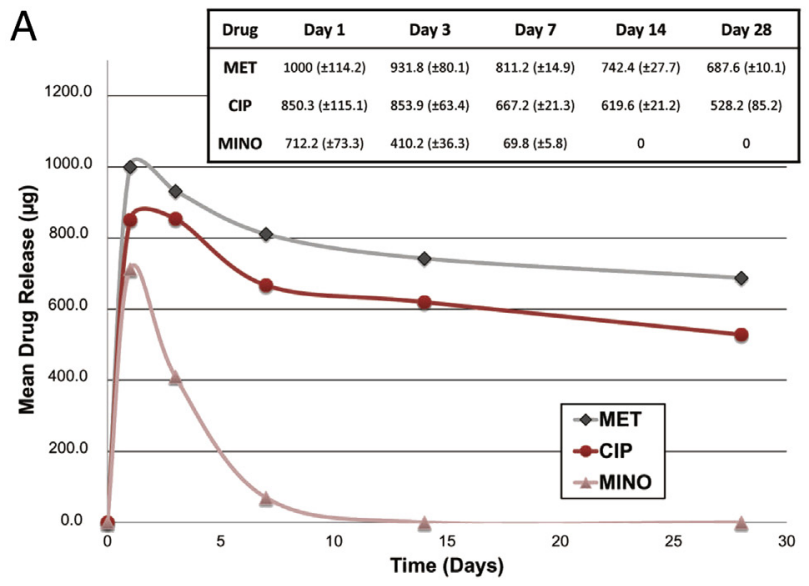

Figure 2. Mean drug release profile $(A)$ and percentage of drug release $(B)$ of TAP-mimic scaffold up to 28 days of incubation in PBS $\left(37^{\circ} \mathrm{C}\right)$. Mean \pm standard deviations of released drugs are presented in the inset table.

\section{Antimicrobial Evaluation of $\boldsymbol{A}$. naes/undit Biofilm-infected Dentin Specimens}

As shown in Table 1, all groups tested were statistically different from each other. TAP solution had the highest percentage of dead bacteria $(P=.0022$ versus TAP-mimic scaffold and $P<.0001$ versus pure PDS and control), followed by the TAP-mimic scaffold $(P<.0001$ versus PDS scaffold and control), which was higher than the control $(P=.0082)$. Figure $3 A$ and $B$ clearly show the presence of $A$. naeslundii biofilm inside the dentinal tubules (green) after 7 days (control group). The formulated TAP solution was able to eliminate all viable bacteria (Table 1 and Fig. $3 D$ ). Whereas for the PDS and control groups the CLSM findings (Fig. $3 A$ and $B$ ) agreed with the SEM images (Fig. $3 \boldsymbol{E}$ and $F$ ) in terms of biofilm formation, the TAP-mimic scaffold was able to kill most of the bacteria (Fig. $3 C$ ), although without removing them from the surface (Fig. 3G). By contrast, TAP solution revealed the formation of insoluble agglomerates over the dentin surface (Fig. $3 D$ and $H$ ). Figure 3 also displays macrophotographs in which both TAP-mimic scaffolds and the TAP solution led to a gray-brownish dentin discoloration, which was significantly more accentuated after TAP-solution exposure (Fig. $3 L$ ). Dentin discoloration was not observed in pure PDS or the negative control (7-day biofilm) (Fig. $3 I$ and $J$ ).

\section{Discussion}

Besides antimicrobial properties, an ideal scaffold to be translated into the clinics for regenerative endodontics must present adequate mechanical properties, geometry, and clinical handleability. One of the key concerns toward the use of scaffolds in endodontics relates to the achievement of an intimate contact within the root canals, avoiding its displacement through the apical foramen and, consequently, the arrest of stem cell proliferation (2). This study investigated some important mechanical properties of a novel TAP-mimic PDS-based scaffold. Our data suggest that the addition of antibiotics led to a significant decrease in tensile strength, mainly after hydration (Fig. $1 E$ ). Antibiotics are usually more hydrophilic than most polymers (eg, polyesters) used to produce electrospun fibers (24-26), which, in turn, may have contributed to the significant decrease in physical stability of the TAPmimic scaffold (26). Although the antibiotics' hydrophilic nature may have contributed to the reduction of TAP-mimic mechanical properties, this characteristic may have positively contributed to the drug release (Fig. 2). All drugs displayed an initial burst release within the first 24 hours, corroborating with previous findings (18), which demonstrated similar MET and CIP release profiles. We also investigated the release profile of MINO. Interestingly, MINO drug concentration was progressively reduced after 24 hours, with no further release after 2 weeks (Fig. 2A). One could speculate that some chemical reactions may have occurred between MINO and one of the compounds used in the HPLC analysis. Indeed, according to Tanase et al (27), MINO may ionize in the acid mobile phase of the system, leading to ion-pair formation and, consequently, to its retention in the solid phase. Future studies will investigate alternative HPLC systems to better evaluate MINO release. Nonetheless, the amount of drugs released from the TAP-mimic scaffold

TABLE 1. Percentage of Live and Dead Cells

\begin{tabular}{|c|c|c|c|c|c|c|c|c|}
\hline Outcome & Groups & No. of samples & No. of areas & Minimum & Maximum & Mean & $\begin{array}{l}\text { SD between } \\
\text { samples }\end{array}$ & $\begin{array}{l}\text { SD within } \\
\text { sample }\end{array}$ \\
\hline \multirow[t]{4}{*}{$\%$ Live } & Control & 4 & 20 & 94.94 & 100.00 & 98.81 & 1.40 & 0.81 \\
\hline & PDS scaffold & 4 & 20 & 91.53 & 98.81 & 95.76 & 1.27 & 1.71 \\
\hline & TAP paste & 4 & 20 & 0.00 & 0.00 & 0.00 & 0.00 & 0.00 \\
\hline & TAP scaffold & 4 & 20 & 0.40 & 6.84 & 2.46 & 0.82 & 2.12 \\
\hline \multirow[t]{4}{*}{$\%$ Dead } & Control & 4 & 20 & 0.00 & 5.06 & 1.19 & 1.40 & 0.81 \\
\hline & PDS scaffold & 4 & 20 & 1.19 & 8.47 & 4.24 & 1.27 & 1.71 \\
\hline & TAP paste & 4 & 20 & 100.00 & 100.00 & 100.00 & 0.00 & 0.00 \\
\hline & TAP scaffold & 4 & 20 & 93.16 & 99.60 & 97.54 & 0.82 & 2.12 \\
\hline \multirow{4}{*}{$\operatorname{Sin}^{-1}\left[(\% \text { dead })^{1 / 2}\right.$} & Control & 4 & 20 & 0.01 & 0.23 & 0.08 & 0.07 & 0.03 \\
\hline & PDS scaffold & 4 & 20 & 0.11 & 0.30 & 0.20 & 0.03 & 0.04 \\
\hline & TAP paste & 4 & 20 & 1.57 & 1.57 & 1.57 & 0.00 & 0.00 \\
\hline & TAP scaffold & 4 & 20 & 1.31 & 1.51 & 1.43 & 0.03 & 0.07 \\
\hline
\end{tabular}

SD, standard deviation. 


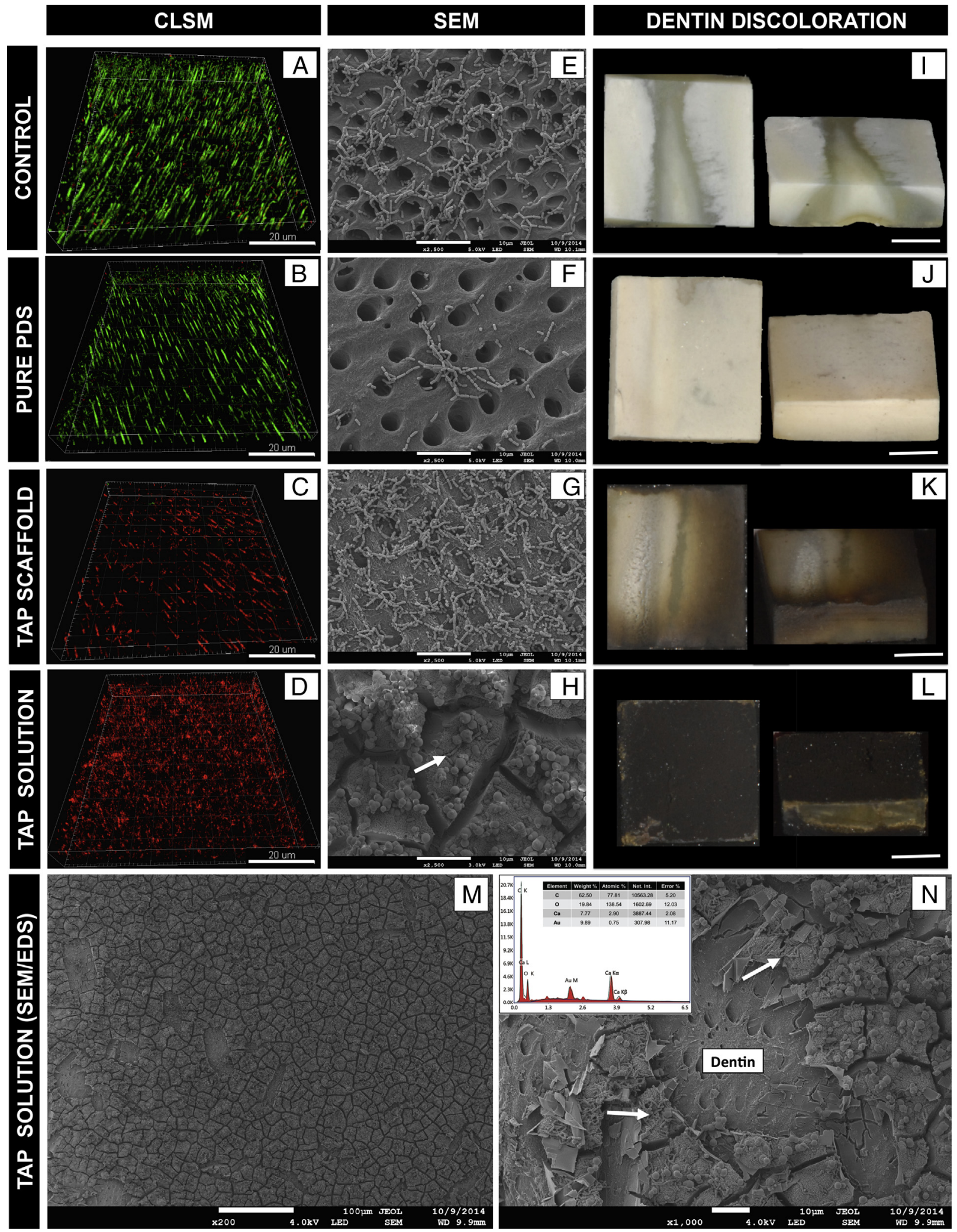

Figure 3. CLSM images were collected in sequential illumination mode by using 488-nm and 552-nm laser lines. Fluorescent emission was collected in 2 HyD spectral detectors with filter range set up to 500-550 nm and 590-655 nm for green (SYTO9) and red dye (PI), respectively. CLSM macrophotographs of 7-day A. naeslundii biofilm (negative control) growth inside dentinal tubules $(A)$, infected dentin treated with pure PDS $(B)$, TAP scaffold $(C)$, and TAP solution $(D)$ for 3 days. SEM images of $A$. naeslundii biofilm on the dentin surface (negative control) $(E)$ treated by pure PDS $(F)$, TAP scaffold $(G)$, and TAP solution $(H)$. Dentin discoloration images of negative control $(I)$, pure PDS $(J)$, TAP scaffold $(K)$, and TAP solution $(L)$ groups. Representative SEM images (original magnification, $\times 200$ and $\times 1000)$ of TAP solution treated dentin showing calcium-enriched $(\mathrm{Ca})$ insoluble agglomerates attached to the dentin surface $(M)$ and covering dentinal tubules $(N)$ as demonstrated by energy-dispersive x-ray spectroscopy (EDS) analyses (inset EDS image $N$ ); $A$. naeslundii can be seen on the surface of this insoluble complex (white arrows) ( $N$ and $H)$. 
throughout the study is well above (Fig. $2 A$ ) the minimum inhibitory concentration for $A$. naeslundii (eg, antibiotic within the tetracycline family such as doxycycline, minimum inhibitory concentration = $0.064 \mu \mathrm{g} / \mathrm{mL})(5)$.

A recent study reported the detection of $A$. naeslundii in initial samples recovered from the infected root canals of necrotic immature permanent teeth that had undergone trauma 2 days to 8 years previously (7). Previous studies have investigated $A$. naeslundii associated with other bacteria, aiming to form multispecies biofilms $(5,28)$. To the best of our knowledge, no attempt has been made to study the influence of time on the formation of $A$. naeslundii biofilm on dentin. Here, a young, 7-day-old $A$. naeslundii biofilm on dentin was developed. Our data revealed numerous bacteria agglomerates fairly well-distributed over the entire dentin surface (SEM), as well as inside the dentinal tubules (CSLM) (Fig. $3 A$ and $E$ ), without the need for special specimen preparation (ie, centrifugation). Importantly, several studies have performed dentin specimen centrifugation to permit the deep penetration of bacteria inside dentinal tubules, simulating longterm infection and to allow for assessment of the antimicrobial effects inside the dentinal tubules (21-23). One can speculate that the adhesion of $A$. naeslundii to dentin surface occurs by means of their unique surface structures, so-called type 1 fimbriae that help them to attach to collagen structures $(29,30)$.

Clinical strategies currently advocated to achieve bacteria/biofilm elimination of the root canal make use of potent chemical substances (eg, TAP), which can be considered extremely toxic to stem cells (4). During the past few years, TAP has been one of the most studied intracanal medicaments as a disinfection step within the regenerative approach. Nonetheless, recent findings have warned about the drawbacks associated with its use (ie, stem cell toxicity (14) and aesthetic issues (31)). Meanwhile, novel antibiotic-containing scaffolds (eg, TAP-mimic scaffolds) have been developed through the wise combination of biodegradable and compatible polymers and considerably lower antibiotic(s) concentration. Notably, the concentration of the 3 antibiotics used to synthesize TAP-mimic scaffolds generally accounts for a few milligrams of drugs (per scaffold), as opposed to the clinically used concentration of TAP paste $(1 \mathrm{~g} / \mathrm{mL})$. Tooth discoloration has been attributed to MINO that acts through the chelation of calcium ions, forming an insoluble complex (27). In this study, dentin discoloration was seen after TAP solution and TAP-mimic scaffold treatments. Moreover, SEM images revealed the formation of calcium-enriched insoluble agglomerates over the dentin surface (27) as demonstrated by energy-dispersive x-ray spectroscopy analyses (Fig. $3 N$, inset). Studies removing or replacing MINO by Augmentin or Cefaclor as intracanal medications have started as potential alternatives to prevent and/ or minimize tooth discoloration $(32,33)$. Indeed, from an antimicrobial viewpoint, this novel TAP-mimic scaffold demonstrated a significant reduction in viable biofilm, even inside dentinal tubules (Fig. 3C). The microbial data obtained after treating the $A$. naeslundii biofilm with TAP-mimic scaffold were similar to those achieved after TAP solution, which carried a considerably higher concentration of antibiotics $(50 \mathrm{mg} / \mathrm{mL}$ of each drug).

Previous studies have demonstrated the antimicrobial effects of both the triple and double antibiotic pastes against Enterococcus faecalis and Porphyromonas gingivalis biofilms $(34,35)$. However, no study has investigated the antimicrobial efficacy of TAP on $A$. naeslundii dentin biofilm. In vitro research has demonstrated that Actinomyces species were resistant to MET but susceptible to moxifloxacin (ie, CIP family) and amoxicillin (5). Therefore, one could certainly argue that both CIP and MINO present in the TAP-mimic scaffold were responsible for most of the antimicrobial action against $A$. naeslundii biofilm. Future studies will be designed to explore the anti- microbial effects of this novel TAP-mimic scaffold against other bacteria as well as a multispecies biofilm before a preclinical animal study is conducted. Moreover, future clinical application of this TAP-mimic scaffold must consider the insertion mechanism, removal, and interference with organic material including fluids and/or blood inside root canals.

Taken together, the novel TAP-mimic scaffolds promoted a significant reduction in A. naeslundii biofilm formed on human dentin. In conjunction with cell-based assays, higher concentrations of the 3 drugs could be proposed to amplify the antimicrobial efficacy without jeopardizing cell compatibility.

\section{Acknowledgments}

The authors thank Mr George J. Eckert for his belp on statistics.

M.C.B. acknowledges funding from an International Development Funds (IDF) Grant from Indiana University Purdue Universityand start-up funds from Indiana University School of Dentistry. This project was supported by the Indiana Clinical and Translational Sciences Institute, funded in part by grant \#UL1 TR001108 from the National Institutes of Health, National Center for Advancing Translational Sciences, Clinical and Translational Sciences Award. The content is solely the responsibility of the authors and does not necessarily represent the official views of the National Institutes of Health.

The authors deny any conflicts of interest related to this study.

\section{References}

1. Diogenes AR, Ruparel NB, Teixeira FB, et al. Translational science in disinfection for regenerative endodontics. J Endod 2014;40:S52-7.

2. Albuquerque MT, Valera MC, Nakashima M, et al. Tissue-engineering-based strategies for regenerative endodontics. J Dent Res 2014;93:1222-31.

3. Xia T, Baumgartner JC. Occurrence of Actinomyces in infections of endodontic origin. J Endod 2003;29:549-52.

4. Al-Ahmad A, Ameen H, Pelz K, et al. Antibiotic resistance and capacity for biofilm formation of different bacteria isolated from endodontic infections associated with root-filled teeth. J Endod 2014;40:223-30.

5. LeCorn DW, Vertucci FJ, Rojas MF, et al. In vitro activity of amoxicillin, clindamycin, doxycycline, metronidazole, and moxifloxacin against oral Actinomyces. J Endod 2007:33:557-60.

6. Chu FC, Leung WK, Tsang PC, et al. Identification of cultivable microorganisms from root canals with apical periodontitis following two-visit endodontic treatment with antibiotics/steroid or calcium hydroxide dressings. J Endod 2006;32:17-23.

7. Nagata JY, Soares AJ, Souza-Filho FJ, et al. Microbial evaluation of traumatized teeth treated with triple antibiotic paste or calcium hydroxide with $2 \%$ chlorhexidine gel in pulp revascularization. J Endod 2014;40:778-83.

8. Distel JW, Hatton JF, Gillespie MJ. Biofilm formation in medicated root canals. J Endod 2002;28:689-93.

9. Ricucci D, Loghin S, Siqueira JF Jr. Exuberant biofilm infection in a lateral canal as the cause of short-term endodontic treatment failure: report of a case. J Endod 2013:39:712-8.

10. Hoshino E, Kurihara-Ando N, Sato I, et al. In-vitro antibacterial susceptibility of bacteria taken from infected root dentine to a mixture of ciprofloxacin, metronidazole and minocycline. Int Endod J 1996;29:125-30.

11. Sato I, Ando-Kurihara N, Kota K, et al. Sterilization of infected root-canal dentine by topical application of a mixture of ciprofloxacin, metronidazole and minocycline in situ. Int Endod J 1996;29:118-24.

12. Banchs F, Trope M. Revascularization of immature permanent teeth with apical periodontitis: new treatment protocol? J Endod 2004;30:196-200.

13. Raju SM, Yadav SS, Kumar MS. Revascularization of immature mandibular premolar with pulpal necrosis: a case report. J Clin Diagn Res 2014;8:ZD29-31.

14. Ruparel NB, Teixeira FB, Ferraz CC, et al. Direct effect of intracanal medicaments on survival of stem cells of the apical papilla. J Endod 2012;38:1372-5.

15. Yadlapati M, Souza LC, Dorn S, et al. Deleterious effect of triple antibiotic paste on human periodontal ligament fibroblasts. Int Endod J 2014;47:769-75.

16. Bottino MC, Kamocki K, Yassen GH, et al. Bioactive nanofibrous scaffolds for regenerative endodontics. J Dent Res 2013;92:963-9.

17. Bottino MC, Yassen GH, Platt JA, et al. A novel three-dimensional scaffold for regenerative endodontics: materials and biological characterizations. J Tissue Eng Regen Med 2013 Mar 8. http://dx.doi.org/10.1002/term.1712 [Epub ahead of print]. 
18. Kamocki K, Nor JE, Bottino MC. Dental pulp stem cell responses to novel antibioticcontaining scaffolds for regenerative. Int Endod J 2014 Nov 25. http://dx.doi.org/ 10.1111/iej.12414 [Epub ahead of print].

19. Albuquerque MT, Valera MC, Moreira CS, et al. Effects of ciprofloxacin-containing scaffolds on Enterococcus faecalis biofilms. J Endod 2015;41:710-4.

20. Wu D, Fan W, Kishen A, et al. Evaluation of the antibacterial efficacy of silver nanoparticles against Enterococcus faecalis biofilm. J Endod 2014;40:285-90.

21. Du T, Wang Z, Shen Y, et al. Effect of long-term exposure to endodontic disinfecting solutions on young and old Enterococcus faecalis biofilms in dentin canals. J Endod 2014;40:509-14.

22. Ma J, Wang Z, Shen $\mathrm{Y}$, et al. A new noninvasive model to study the effectiveness of dentin disinfection by using confocal laser scanning microscopy. J Endod 2011;37: 1380-5.

23. Wang Z, Shen Y, Haapasalo M. Effectiveness of endodontic disinfecting solutions against young and old Enterococcus faecalis biofilms in dentin canals. J Endod 2012;38:1376-9.

24. Kim K, Luu YK, Chang C, et al. Incorporation and controlled release of a hydrophilic antibiotic using poly(lactide-co-glycolide)-based electrospun nanofibrous scaffolds. J Control Release 2004;98:47-56.

25. Liang D, Hsiao BS, Chu B. Functional electrospun nanofibrous scaffolds for biomedical applications. Adv Drug Deliv Rev 2007;59:1392-412.

26. Feng K, Sun H, Bradley MA, et al. Novel antibacterial nanofibrous PLLA scaffolds. J Control Release 2010;146:363-9.
27. Tanase S, Tsuchiya H, Yao J, et al. Reversed-phase ion-pair chromatographic analysis of tetracycline antibiotics: application to discolored teeth. J Chromatogr B Biomed Sci Appl 1998;706:279-85.

28. Bergmans L, Moisiadis P, Teughels W, et al. Bactericidal effect of Nd:YAG laser irradiation on some endodontic pathogens ex vivo. Int Endod J 2006;39:547-57.

29. Tang G, Yip HK, Samaranayake LP, et al. Direct detection of cell surface interactive forces of sessile, fimbriated and non-fimbriated Actinomyces spp. using atomic force microscopy. Arch Oral Biol 2004;49:727-38.

30. Liu T, Gibbons RJ, Hay DI, et al. Binding of Actinomyces viscosus to collagen: association with the type 1 fimbrial adhesin. Oral Microbiol Immunol 1991;6:1-5.

31. Kim JH, Kim Y, Shin SJ, et al. Tooth discoloration of immature permanent incisor associated with triple antibiotic therapy: a case report. J Endod 2010;36:1086-91.

32. Akcay M, Arslan H, Yasa B, et al. Spectrophotometric analysis of crown discoloration induced by various antibiotic pastes used in revascularization. J Endod 2014;40:845-8.

33. Bezgin T, Yilmaz AD, Celik BN, et al. Efficacy of platelet-rich plasma as a scaffold in regenerative endodontic treatment. J Endod 2015;41:36-44.

34. Sabrah AH, Yassen GH, Gregory RL. Effectiveness of antibiotic medicaments against biofilm formation of Enterococcus faecalis and Porphyromonas gingivalis. J Endod 2013;39:1385-9.

35. Ordinola-Zapata R, Bramante CM, Minotti PG, et al. Antimicrobial activity of triantibiotic paste, $2 \%$ chlorhexidine gel, and calcium hydroxide on an intraoral-infected dentin biofilm model. J Endod 2013;39:115-8. 